\title{
MILP branch flow model for concurrent AC multistage transmission expansion and reactive power planning with security constraints
}

ISSN 1751-8687

Received on 19th January 2016 Revised on 7th April 2016 Accepted on 14th May 2016 doi: 10.1049/iet-gtd.2016.0081 www.ietdl.org

\section{Leonardo H. Macedo ${ }^{1}$, Cristiam V. Montes ${ }^{1}$, John F. Franco ${ }^{1}$, Marcos J. Rider ${ }^{凶}$, Rubén Romero ${ }^{1}$ \\ ${ }^{1}$ Department of Electrical Engineering, São Paulo State University, PO Box 31, Av. Brasil Centro 56, 15385-000, Ilha Solteira-SP, Brazil ${ }^{2}$ Department of Systems and Energy, University of Campinas, Av. Albert Einstein 400, 13083-852, Campinas-SP, Brazil \\ $\bowtie$ E-mail: mjrider@dsee.fee.unicamp.br}

\begin{abstract}
This study presents a mixed-integer linear programming (MILP) model to solve the simultaneous transmission network expansion planning (TNEP) and reactive power planning (RPP) problem. The proposed model considers reactive power, off-nominal bus voltage magnitudes, power losses, multistage expansion, and security constraints. The use of an MILP model guarantees convergence to optimality by using existing classical optimisation methods. In order to validate the approximation performed, the steady-state operation points were compared with those obtained using an AC load flow method. Garver's 6-bus system and a modified IEEE 118-bus system were used to show the precision and efficiency of the methodology. The results indicate that better expansion and generation plans are found by considering RPP simultaneously with the AC TNEP, when the solutions were compared with the plans of the TNEP using the AC model without RPP and the TNEP considering the DC model, with RPP conducted at a subsequent stage.
\end{abstract}

\author{
Nomenclature \\ Sets \\ $\Omega \quad$ set of transmission corridors \\ $K \quad$ set of system conditions, i.e. normal condition $\left(K^{0}\right)$, \\ contingencies in existing or candidate lines $\left(K^{1}\right)$ \\ $N \quad$ set of buses \\ $R \quad$ set of candidate $\operatorname{VAr}$ sources \\ $T \quad$ set of stages \\ $Y \quad$ set of equivalents of the candidate lines
}

\section{Functions}

$\mathrm{CE}_{t}, \mathrm{CO}_{t} \quad$ costs of expansion and operation for stage $t$

\section{Constants}

\section{$\alpha \quad$ annual discount rate}

$\Delta_{\kappa} \quad$ annual system operation time in condition $\kappa$

$\overline{\Delta S}_{i j, y, \kappa} \quad$ upper bound of each block of the power flow

linearisation of corridor $i j$, equivalent line $y$,

condition $\kappa$

$\psi_{t} \quad$ load factor for stage $t$

$\Gamma_{i j, \kappa} \quad$ state of an existing line or candidate line in corridor

$\bar{\theta} \quad$ maximum voltage phase angle difference

$v_{i, t, \kappa} \quad$ value of the voltage magnitude at bus $i$, at stage $t$, in

condition $\kappa$, estimated from the relaxed LP model

$Y_{t}^{i}, Y_{t}^{f} \quad$ initial and final operation year of stage $t$

$B^{\text {shc }}$

$b_{i j, y, \kappa}^{\text {shl }}$

$c_{i}^{\mathrm{g}}$

$c_{i j, y}^{1}$

$c_{i}^{\mathrm{sh}}$
$\bar{I}_{i j, y, \kappa}$

$L$

$\bar{m}_{i}$

$m_{i j, y, l, \kappa}^{\mathrm{S}}$

$n_{i j, y, \kappa}$

$\bar{n}_{i j}$

$P_{i, t}^{\mathrm{d}}, Q_{i, t}^{\mathrm{d}}$

$\bar{P}_{i, t}^{\mathrm{g}}, \underline{P}_{i, t}^{\mathrm{g}}$

$\bar{Q}_{i, t}^{\mathrm{g}}, \underline{Q}_{i, t}^{\mathrm{g}}$

$r_{i j, y, \kappa}, x_{i j, y, \kappa}$

$S^{\mathrm{b}}$

$\bar{V}, \underline{V}$

$V^{\mathrm{n}}-$

$z_{i j, y, \kappa}$

maximum current flow in equivalent line $y$ in corridor $i j$ and condition $\kappa$

number of blocks of the piecewise linearisation maximum number of candidate VAr sources at bus $i$ slope of the $l$ th block used in the linearisation of the power flow of corridor $i j$, equivalent line $y$, and condition $\kappa$

number of lines in corridor $i j$, equivalent $y$, and condition $\kappa$ maximum number of candidate lines in corridor $i j$ active and reactive power demands at bus $i$, stage $t$ maximum and minimum limits of active power generation at bus $i$ at stage $t$ maximum and minimum limits of reactive power generation at bus $i$ at stage $t$ resistance and reactance of equivalent line $y$ in corridor $i j$ and condition $\kappa$

apparent power base

maximum and minimum voltage magnitudes nominal voltage magnitude of the system impedance of equivalent line $y$ in corridor $i j$ and condition $\kappa$

\section{Continuous variables}

$\Delta P_{i j, y, l, t, \kappa}, \Delta Q_{i j, y, l, t, \kappa}$

$f_{i j, t, \kappa}^{\theta}$

shunt susceptance of equivalent line $y$ in corridor $i j$ and condition $\kappa$

cost of generation of generator at bus $i$

cost of equivalent line $y$ in corridor $i j$

cost of a VAr source that can be added at bus $i$ $\theta_{i, t, \kappa}$

$f_{i j, t, \kappa}^{\mathrm{V}}$

$I_{i j, y, t, \kappa}$ values of the $l$ th block associated with active and reactive power flows in corridor $i j$, equivalent line $y$, at stage $t$, in condition $\kappa$ voltage phase angle at bus $i$, at stage $t$, in condition $\kappa$

slack variable of the voltage phase angle calculation equation of corridor $i j$, at stage $t$, in condition $\kappa$

slack variable of the voltage drop equation of corridor $i j$, at stage $t$, in condition $\kappa$ current flow magnitude on equivalent line $y$, in corridor $i j$, at stage $t$, in condition $\kappa$ 


\begin{tabular}{|c|c|}
\hline$I_{i j, y, t, \kappa}^{\mathrm{sqr}}$ & square of $I_{i j, y, t, \kappa}$ \\
\hline$P_{i j, y, t, \kappa}$ & active and reactive power flows in \\
\hline$Q_{i j, y, t, \kappa}$ & $\begin{array}{l}\text { equivalent line } y \text { in corridor } i j \text {, at stage } t \text {, in } \\
\text { condition } \kappa\end{array}$ \\
\hline$P_{i, t, \kappa}^{\mathrm{g}}, Q_{i, t, \kappa}^{\mathrm{g}}$ & $\begin{array}{l}\text { active and reactive power generations at bus } \\
i \text { at stage } t \text { in condition } \kappa\end{array}$ \\
\hline$P_{i j, y, t, \kappa}^{+}, P_{i j, y, t, \kappa}^{-}$ & positive variables in the calculation of $\left|P_{i j, y, t, \kappa}\right|$ \\
\hline$Q_{i, r, t, \kappa}^{\mathrm{shc}}$ & $\begin{array}{l}\text { reactive power injection by the candidate } \\
\text { VAr source } r \text {, at bus } i \text {, at stage } t \text {, in } \\
\text { condition } \kappa\end{array}$ \\
\hline$Q_{i, i j, y, t, \kappa}^{\mathrm{shl}}$ & $\begin{array}{l}\text { reactive power injection at bus } i \text { by the } \\
\text { shunt of equivalent line } y \text { in corridor } i j \text {, at } \\
\text { stage } t \text {, condition } \kappa\end{array}$ \\
\hline$Q_{i j, y, t, \kappa}^{+}, Q_{i j, y, t, \kappa}^{-}$ & positive variables in the calculation of $\mid Q_{i j, y}$, \\
\hline$V_{i, t, \kappa}$ & $\begin{array}{l}\text { voltage magnitude at bus } i \text {, at stage } t \text {, in } \\
\text { condition } \kappa\end{array}$ \\
\hline$V_{i, t, \kappa}^{\mathrm{sqr}}$ & square of $V_{i, t, \kappa}$ \\
\hline
\end{tabular}

\section{Binary variables}

$h_{i, r, t} \quad$ installation of VAr source $r$ at bus $i$ at stage $t$

$w_{i j, y, t} \quad$ construction of equivalent line $y$ in corridor $i j$ at stage $t$

\section{Introduction}

The transmission network expansion planning (TNEP) problem aims at determining the location, number, and timing of new transmission lines, transformers, and other devices to be added to the transmission network in order to properly meet future power demand with minimum investment cost [1]. The most widely used approach to solve the TNEP problem considers a simplified system model (with various degrees of approximation) in the first phase of the planning, for example the transportation model, the hybrid model, the DC model, and the linear disjunctive model [2]. The solution techniques for this first phase include classical optimisation methods and metaheuristics, such as tabu search, ant colony, greedy randomised search, particle swarm optimisation, and genetic algorithms. The expansion plan obtained in the first phase is then reinforced in a second phase through reactive power planning (RPP), and voltage and transient stability analyses [3]. In the first phase, the TNEP problem can be classified as static when it is a single-stage problem, or as multistage when the planning horizon is divided into several stages. Since the load in the future cannot be accurately known, a more suitable model considers uncertainty in demand. In addition, contingency criteria can be considered in TNEP. In [4], works that consider planning with security constraints, uncertainty in demand, and static/multistage planning are presented. The RPP problem is usually solved after the TNEP, since only active power is considered in the TNEP problem using the DC model in the first phase. The main objective of the RPP problem is to determine the minimal cost of shunt reactive compensators, which must be connected to each bus so that the system operation is appropriate. A complete survey on RPP can be found in [5]. Although most studies consider the DC model in the first phase of the TNEP, there are some issues with this approach, as presented in [6,7]: (i) the TNEP problem must be treated separately from the RPP problem; (ii) it is frequently necessary to reinforce an expansion plan obtained using the DC model when an operation with the AC model is considered; and (iii) it is difficult to take into account the power losses, reactive power flows, and off-nominal bus voltage magnitudes. Furthermore, the results with the DC power flow may differ considerably from the results with the AC load flow [7].

As $[6,7]$ demonstrate, the RPP must be considered simultaneously when using the AC model in the TNEP (ACTNEP). Without considering VAr source allocation, the solution of the TNEP problem will only have generators and line shunts providing the reactive power demanded by the loads. Because of this, the plan may require more transmission lines to be added, leading to low-quality solutions [7]. In addition, other components (such as flexible alternating current transmission system devices) can be included, and other types of studies (such as voltage stability, nodal analysis, transient stability analysis, etc.) can be carried out in the ACTNEP. Moreover, the difficulties that appear when working with the ACTNEP are: (i) handling disconnected systems, a common situation in the initial phase of TNEP when generators and loads have not yet been electrically connected to the network; (ii) developing an efficient optimisation technique; and (iii) working with large and complex mixed-integer non-linear programming (MINLP) problems. In particular, the problem described in (i) may appear when heuristic methodologies are used to solve the TNEP problem, hence strategies must be defined to avoid load shedding. When an exact model is used, as the one proposed in this paper, disconnected systems are not an issue, since the investment variables will ensure the feasible operation of the system (by installing sufficient lines); in addition, disconnected buses do not increase the complexity of the problem.

Over the past decade, there has been growing attention paid to ACTNEP. The works in [7-11] use metaheuristic or heuristic techniques to solve the ACTNEP problem with RPP. Although these techniques are robust, flexible, and achieve good results, they present many problems, such as high computational demand, the adjustment and tuning of parameters, and the definition of a stop criterion. In addition, they cannot guarantee convergence to the global optimum [12]. The authors in [13-19] propose approximate and relaxed models to solve the ACTNEP problem using exact methods. Zhang et al. [13] proposes two non-linear programming relaxations for the original MINLP problem, and solves the relaxed and the original models in order to compare the results. In [14], a mixed-integer linear programming (MILP) model is proposed to solve the static ACTNEP problem, according to an iterative procedure that calculates the exact power flow to determine the system reinforcements for normal and contingency operation. Akbari and Bina [15] proposes an MILP model for the multistage problem; in [16] an improvement to the traditional DC TNEP model and a linearised ACTNEP model are presented; Asadamongkol and Eua-Arporn [17] proposes a Benders decomposition procedure for the ACTNEP problem, employing RPP after line expansion planning. Finally, Taylor and Hover [18] and Jabr [19] present mixed-integer conic programming formulations for the problem. Although these works propose more realistic models, only Jabr [19] considers RPP simultaneously. Furthermore, none of these studies presents an analysis of the accuracy of the results obtained.

This work presents a novel MILP model for solving the TNEP problem simultaneously with RPP, considering a new linearised AC model of the network operation. The model for the TNEP also considers multistage expansion and security constraints. The use of an MILP model guarantees optimality by using classical optimisation tools. The proposed methodology was implemented using the algebraic modelling language AMPL [20], and solutions were found via the commercial optimisation solver CPLEX [21]. In order to validate the approximation performed, the steady-state operation points were compared with those obtained using an $\mathrm{AC}$ load flow method. Garver's 6-bus system and a modified IEEE 118-bus system were used to prove the accuracy of the TNEP model. The main contribution of this paper is a new MILP model for ACTNEP with simultaneous RPP considering multistage expansion and security constraints. This model is based on the branch flow formulation for the AC load flow [22]. The results obtained demonstrate that the proposed model can provide solutions that are feasible for the original constraints of the ACTNEP problem, differently from existing DC and ACTNEP models [18].

\section{Model for AC load flow}

Fig. 1 illustrates two branches of a transmission network (TN), connecting buses $i, j$, and $k$. In this paper, the transmission lines are represented using the $\pi$ model, which is suitable for lines 


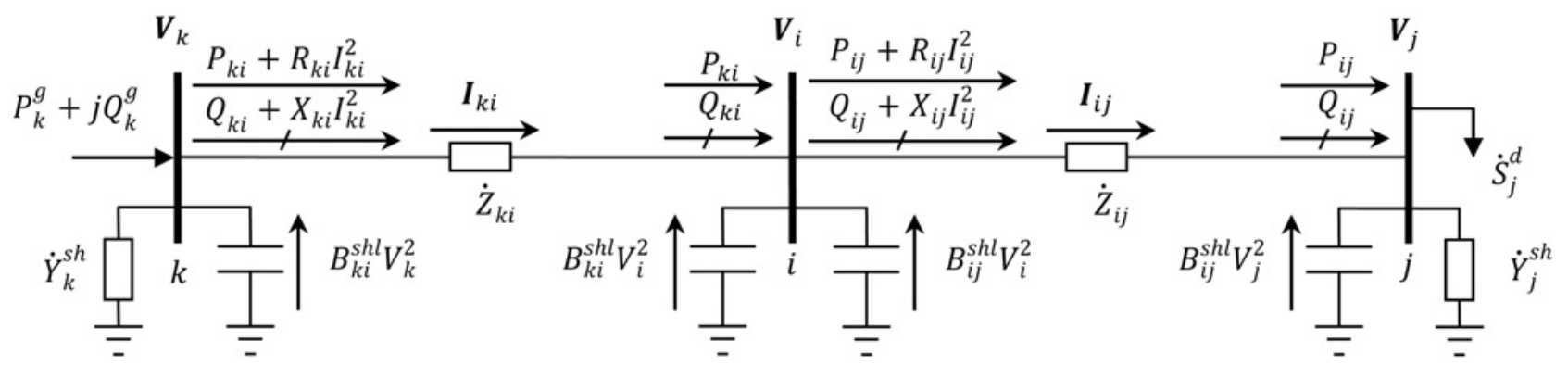

Fig. 1 Illustrative transmission network

$<240 \mathrm{~km}$ long [23]. The quantities $\boldsymbol{V}_{i}$ and $\boldsymbol{I}_{i j}$ are the phasors of the voltage at bus $i$ and the current flow on branch $i j$, respectively. $P_{i j}$ and $Q_{i j}$ are the active and reactive power through the series impedance of branch $i j$ arriving at bus $j$, while $P_{i j}+R_{i j} I_{i j}^{2}$ is the active power through the series impedance of branch $i j$ leaving bus $i$. The parameters $\dot{Z}_{i j}=R_{i j}+j X_{i j}$ and $B_{i j}^{\text {shl }}$ are the complex impedance of branch $i j$ and half of the total line $i j$ charging. $\dot{Y}_{i}^{\mathrm{sh}}=G_{i}^{\mathrm{sh}}+j B_{i}^{\mathrm{sh}}$ is the shunt admittance connected to bus $i$. $\dot{S}_{j}^{\mathrm{d}}=P_{j}^{\mathrm{d}}+j Q_{j}^{\mathrm{d}}$ is the complex power demand at bus $j . R_{i j} I_{i j}^{2}$ and $X_{i j} I_{i j}^{2}$ are the active and reactive power losses of branch $i j$, respectively. It is assumed that each branch is identified in a unique way, i.e. active power in branch $i j$ is defined using the variable $P_{i j}$ and there is not a variable $P_{j i}$; the active power injection at bus $j$, related to branch $i j$, is defined as $-\left(P_{i j}+R_{i j} I_{i j}^{2}\right)$.

The voltage drop in branch $i j$ in Fig. 1 is represented by

$$
\boldsymbol{V}_{i}-\boldsymbol{V}_{j}=\boldsymbol{I}_{i j}\left(R_{i j}+j X_{i j}\right) \quad \forall i j \in \Omega
$$

where $\boldsymbol{I}_{i j}$ can be calculated using the following equation

$$
\boldsymbol{I}_{i j}=\left(\frac{P_{i j}+j Q_{i j}}{\boldsymbol{V}_{j}}\right)^{*} \quad \forall i j \in \Omega
$$

Equation (2) is then placed in (1) to obtain the following equation

$$
\left(\boldsymbol{V}_{i}-\boldsymbol{V}_{j}\right) \boldsymbol{V}_{j}^{*}=\left(P_{i j}-j Q_{i j}\right)\left(R_{i j}+j X_{i j}\right) \quad \forall i j \in \Omega
$$

Considering $\boldsymbol{V}_{i}=V_{i} \angle \theta_{i}, \boldsymbol{V}_{j}=V_{j} \angle \theta_{j}$, and $\theta_{i j}=\theta_{i}-\theta_{j}$, where $\theta_{i}$ is the voltage phase angle at bus $i$, (3) can be written as.

$$
V_{i} V_{j}\left(\cos \theta_{i j}+j \sin \theta_{i j}\right)-V_{j}^{2}=\left(P_{i j}-j Q_{i j}\right)\left(R_{i j}+j X_{i j}\right) \quad \forall i j \in \Omega
$$

Identifying the real and imaginary parts of (4), the following equations are obtained:

$$
\begin{gathered}
V_{i} V_{j} \cos \theta_{i j}=V_{j}^{2}+R_{i j} P_{i j}+X_{i j} Q_{i j} \quad \forall i j \in \Omega \\
V_{i} V_{j} \sin \theta_{i j}=X_{i j} P_{i j}-R_{i j} Q_{i j} \quad \forall i j \in \Omega
\end{gathered}
$$

Summing the squares of (5) and (6), we get:

$$
V_{i}^{2}-V_{j}^{2}=2\left(R_{i j} P_{i j}+X_{i j} Q_{i j}\right)+Z_{i j}^{2} I_{i j}^{2} \quad \forall i j \in \Omega
$$

where the current flow magnitude $I_{i j}$ is calculated as

$$
I_{i j}^{2}=\frac{P_{i j}^{2}+Q_{i j}^{2}}{V_{j}^{2}} \quad \forall i j \in \Omega
$$

Note that, from (6) and (7), it is possible to obtain the voltage and the phase angle difference across branch $i j$. The systematic application of Kirchhoff's voltage law to the set of fundamental loops of the system is represented in (6)-(8). Note that (5) and (6) can also fully describe the application of Kirchhoff's voltage law to the set of fundamental loops. Equations (7) and (8) are derived to facilitate the development of a linear model in this paper. When radial systems are considered, as in [24], (7) and (8) fully represent the application of Kirchhoff's voltage law to the system, since the voltage angles do not play an important role in radial systems. However, in meshed systems, the voltage angles must be considered, and, therefore, (5) or (6) must be considered together with (7) and (8). In this paper, (6) is chosen to facilitate the linearisation that will be presented.

The conventional equations of load balance (Kirchhoff's current law) are shown as

$$
P_{i}^{\mathrm{g}}-P_{i}^{\mathrm{d}}-G_{i}^{\mathrm{sh}} V_{i}^{2}+\sum_{k i \in \Omega} P_{k i}-\sum_{i j \in \Omega}\left(P_{i j}+R_{i j} I_{i j}^{2}\right)=0 \quad \forall i \in N
$$

$$
\begin{aligned}
Q_{i}^{\mathrm{g}}- & Q_{i}^{\mathrm{d}}+B_{i}^{\mathrm{sh}} V_{i}^{2}+\sum_{k i \in \Omega}\left(Q_{k i}+B_{k i}^{\mathrm{shl}} V_{i}^{2}\right) \\
& -\sum_{i j \in \Omega}\left(Q_{i j}-B_{i j}^{\mathrm{shl}} V_{i}^{2}+X_{i j} I_{i j}^{2}\right)=0 \quad \forall i \in N
\end{aligned}
$$

In (9), $G_{i}^{\text {sh }} V_{i}^{2}$ denotes the active power of the shunt admittance connected to bus $i$. Since this admittance extracts power from the system, it is considered to have a negative sign in this equation. The summation term for $P_{k i}$ represents the total active power arriving at bus $i$ from the branches connected to this bus, while the summation term for $P_{i j}+R_{i j} I_{i j}^{2}$ represents the total active power leaving bus $i$ through branches connected to this bus, considering the active power losses.

In (10), $B_{i}^{\text {sh }} V_{i}^{2}$ represents the reactive power of the shunt susceptance connected to bus $i$. When the shunt element has a capacitive characteristic, $B_{i}^{\text {sh }}>0$, and reactive power is injected in the system. If the shunt susceptance has an inductive characteristic, $B_{i}^{\text {sh }}<0$, and reactive power is absorbed from the system. The shunt elements representing the line charging of the $\pi$ model must be considered at both sides of each line so their contributions are added to both summation terms of (10) (see Fig. 1).

With $\theta_{i}=0$ fixed for the slack bus, (6)-(10) represent the steady-state operation of the $\mathrm{TN}$, where phase shifters and off-nominal transformer turns ratios are not considered. For the power flow calculation, voltage magnitudes of the PV buses and the slack bus must be fixed according to the specified values, and the active power generation must be fixed for the PV buses. For an optimal power flow calculation, only the reference voltage phase angle must be fixed, and the limits of the variables should be considered. An objective function that minimises the cost of active power generation or the losses in the system may be used according to the considered problem.

The model presented in this section is an extension of the branch flow proposal presented in $[22,24]$, where line shunts are not considered and constraint (6) does not need to appear in the 
model, since the voltage phase angle can be eliminated from the formulation.

\section{MILP model for the TNEP with RPP}

This section presents the complete MILP model for the ACTNEP problem with simultaneous RPP based on the formulation presented in the previous section.

The model considers a planning horizon divided into stages, the construction of lines and reactive sources, and contingencies in existing and new lines that may be constructed.

\subsection{Line parameters}

Fig. 2 illustrates the possible line installation options $y$ in a transmission corridor $i j$. A binary variable $w_{i j, y, t}$ indicates which option is chosen for each stage $t$.

For each corridor, the value of $y$ indicates the number of new lines in each option, i.e. $y=0$ means that no line is constructed, $y=1$ means that one new line is constructed and so on. Note that, unlike in works that consider the sequential addition of lines, in this formulation, only one option $y$ must be chosen for corridor $i j$ at stage $t$. Parameter $n_{i j, y, \kappa}=\max \left\{0, n_{i j}^{0}+y-\Gamma_{i j, \kappa}\right\}$ is defined to represent the number of lines in corridor $i j$, option $y$, and condition $\kappa$, where $n_{i j}^{0}$ indicates the number of existing lines in corridor $i j$, and $\Gamma_{i j, \kappa}$ indicates in which corridor $i j$ contingency $\kappa$ occurs, i.e. $\Gamma$ is a $|\Omega| \times|K|$ matrix in which each row indicates a corridor and each column a contingency. In the column of $\Gamma$ corresponding to $\kappa=0$, all entries are zero; in the other columns, only the entry corresponding to the line with the contingency is equal to one, whereas the other elements are zero.

To illustrate the definition of the parameter $n_{i j, y, \kappa}$, let us consider the following two situations for a generic corridor $i j$, for which three new lines can be added: (i) there is no existing line in this corridor, $n_{i j}^{0}=0$; and (ii) there is one existing line in this corridor, $n_{i j}^{0}=1$. For these situations, first consider that in contingency scenario $\kappa$ there is not an outage of a line in corridor $i j\left(\Gamma_{i j, \kappa}=0\right)$. Therefore, in situation (i), $n_{i j, 0, \kappa}=0, n_{i j, 1, \kappa}=1, n_{i j, 2, \kappa}=2$, and $n_{i j, 3, \kappa}$ $=3$, indicating that the number of lines in the corridor is equal to the number of the new constructed lines; and in situation (ii), $n_{i j, 0, \kappa}=1, n_{i j, 1, \kappa}=2, n_{i j, 2, \kappa}=3$, and $n_{i j, 3, \kappa}=4$, indicating that the number of lines in the corridor is equal to the number of the new constructed lines plus the existing line. Now, considering that in contingency scenario $\kappa$ there is an outage of a line in corridor $i j$ ( $\Gamma_{i j, \kappa}=1$ ), situation (i) will have $n_{i j, 0, \kappa}=0, n_{i j, 1, \kappa}=0, n_{i j, 2, \kappa}=1$, and $n_{i j, 3, \kappa}=2$, indicating that if no line is constructed, the number of lines will be equal to zero; if a new line is constructed, an outage is considered, hence the number of lines will also be equal to zero; if two lines are constructed, the number of lines after the outage will be equal to one; and if three lines are constructed, after the

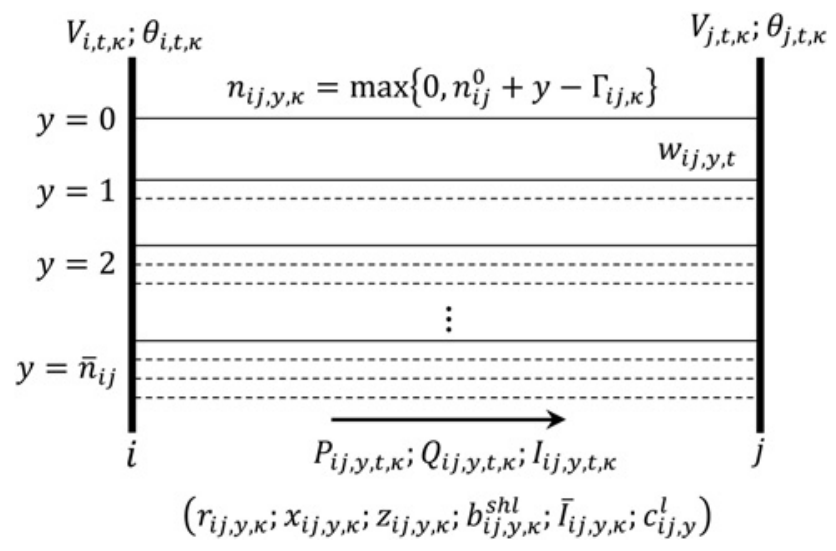

Fig. 2 Line installation options in a transmission corridor outage, there will be two lines in this corridor. This situation represents the contingency case in new lines. For situation (ii), considering that in contingency scenario $\kappa$ there is an outage of a line in corridor $i j\left(\Gamma_{i j, \kappa}=1\right)$, since there is an existing line in corridor $i j$, the contingency in this corridor will represent, without loss of generality, the outage of this existing line, hence the values of $n_{i j, y, \kappa}$ are as follows: $n_{i j, 0, \kappa}=0, n_{i j, 1, \kappa}=1, n_{i j, 2, \kappa}=2$, and $n_{i j, 3, \kappa}=3$.

The parameters of the lines in each corridor $i j$, i.e. $R_{i j}, X_{i j}, B_{i j}^{\text {shl }}$, maximum current flows in normal and contingency conditions, $\bar{I}_{i j}$ and $\overline{\bar{I}}_{i j}$, and $C_{i j}^{\mathrm{l}}$ (the cost of a new line in corridor $i j$ ), must be redefined in terms of each equivalent in option $y$ and contingency scenario $\kappa$. Moreover, the variables that represent the active and reactive power flows and currents in each corridor $i j$ must be redefined for each option $y$, stage $t$, and condition $\kappa$. Since the cost of choosing one option $y$ does not depend on the contingency scenario, it can be defined for equivalent $y$ in corridor $i j$ as $c_{i j, y}^{1}=y \cdot C_{i j}^{\mathrm{l}}$. The maximum current under normal conditions, maximum current under contingency conditions, and the shunt susceptance of equivalent line $y$ in corridor $i j$ in condition $\kappa$ are $\bar{I}_{i j, y, \kappa}=n_{i j, y, \kappa} \cdot \bar{I}_{i j}, \quad \forall \kappa \in K^{0} ; \bar{I}_{i j, y, \kappa}=n_{i j, y, \kappa} \cdot \overline{\bar{I}}_{i j}, \quad \forall \kappa \in K^{1} ; \quad$ and $b_{i j, y, \kappa}^{\text {shl }}=n_{i j, y, \kappa} \cdot B_{i j}^{\text {shl }}$. For $n_{i j, y, \kappa}>0$, the equivalent resistance and reactance of each option $y$ in corridor $i j$ in condition $\kappa$ can be defined as $r_{i j, y, \kappa}=R_{i j} / n_{i j, y, \kappa}$ and $x_{i j, y, \kappa}=X_{i j} / n_{i j, y, \kappa}$. If $n_{i j, y, \kappa}=0$, they can be defined as $r_{i j, y, \kappa}=0$ and $x_{i j, y, \kappa}=0$, and the variables $P_{i j, y, t, \kappa}$, $Q_{i j, y, t, \kappa}$, and $I_{i j, y, t, \kappa}^{\mathrm{sqr}}$ can be fixed at zero. The square value of the magnitude of impedance of the equivalent line of option $y$ in corridor $i j$ in scenario $\kappa$ is $z_{i j, y, \kappa}^{2}=r_{i j, y, \kappa}^{2}+x_{i j, y, \kappa}^{2}$. In this paper, it is considered that all of the lines in the same corridor have the same parameters. To consider lines with different parameters in the same corridor, the equivalent parameters for each option $y$ must be calculated considering these different values.

\subsection{Reactive compensation}

Fig. 3 shows a bus in the system where a shunt element $B_{i}^{\text {sh }}$ is already installed. The installation of VAr source $r$ with susceptance $B^{\text {shc }}$ and reactive power injection $Q_{i, r, t, \kappa}^{\text {shc }}$ at bus $i$ at stage $t$ is represented by the binary variable $h_{i, r, t}$. Note that the investment variable does not depend on condition $\kappa$.

\subsection{Objective function}

The objective in this work is to jointly minimise the costs of expansion and operation, as considered in [14] for the static TNEP without RPP. The cost of expansion consists of the installation of transmission lines and VAr compensators. Since the planning horizon is divided in multistage expansion, the investment cost $\mathrm{CE}_{t}$ for each stage $t$ must be discounted back to its present value. Fig. $4 a$ shows a planning horizon with three stages of five years each.

An annual discount rate of $\alpha$ is assumed. The operation for a stage considers the topology of the system planned in the previous stage. Fig. $4 b$ shows the operation timeline, where the cost of operation $\mathrm{CO}_{t}$ is the discounted value for stage $t$, which corresponds to the time interval $Y_{t}^{i}$ until $\Upsilon_{t}^{f}$, so that both the costs of expansion and

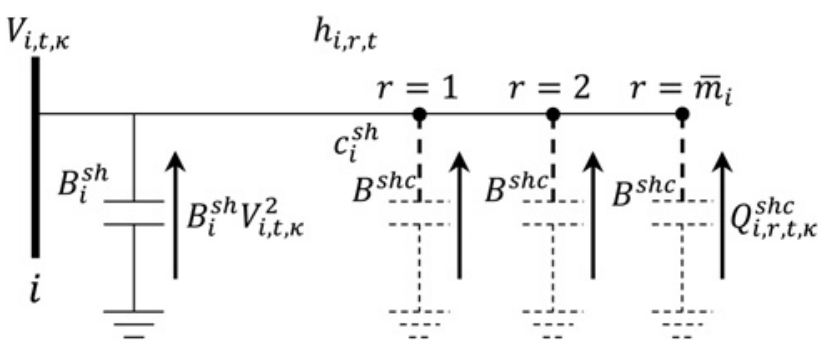

Fig. 3 Reactive shunt compensation installation 


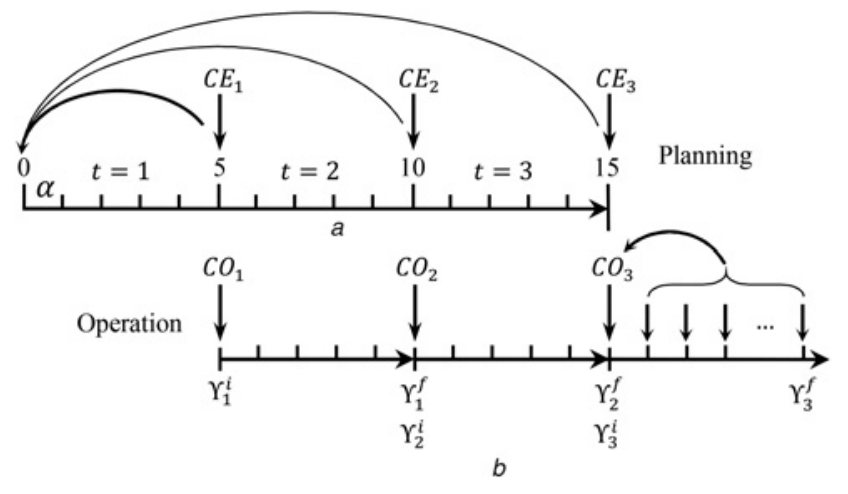

Fig. 4 Planning horizon with three stages of five years each and operation timeline

$a$ Planning timeline

$b$ Operation timeline

operation may be discounted back to their present value as

$$
\min \sum_{t \in T} \frac{1}{(1+\alpha)^{Y_{t}^{i}}}\left(\mathrm{CE}_{t}+\mathrm{CO}_{t}\right)
$$

The cost of expansion in stage $t, \mathrm{CE}_{t}$, is shown in (12) for the first stage and in (13) for $t>1$.

$$
\begin{gathered}
\mathrm{CE}_{t \mid t=1}=\sum_{i j \in \Omega} \sum_{y \in Y} c_{i j, y}^{1} w_{i j, y, 1}+\sum_{i \in N} \sum_{r \in R} c_{i}^{\mathrm{sh}} h_{i, r, 1} \\
\mathrm{CE}_{t \mid t>1}=\sum_{i j \in \Omega} \sum_{y \in Y} c_{i j, y}^{1}\left(w_{i j, y, t}-w_{i j, y, t-1}\right)+\sum_{i \in N} \sum_{r \in R} c_{i}^{\mathrm{sh}}\left(h_{i, r, t}-h_{i, r, t-1}\right)
\end{gathered}
$$

The cost of operation for the period $\Upsilon_{t}^{i}$ until $Y_{t}^{f}$, discounted back to the end of planning stage $t, \mathrm{CO}_{t}$, is shown in (14) [25]. It is assumed that the demand is constant during an operation period.

$$
\mathrm{CO}_{t}=\left(\frac{\psi_{t} \cdot S^{\mathrm{b}}}{10^{6}}\right)\left(\frac{(1+\alpha)^{\mathrm{Y}_{t}^{f}-\Upsilon_{t}^{i}}-1}{\alpha(1+\alpha)^{\mathrm{Y}_{t}^{f}-\Upsilon_{t}^{i}}}\right) \sum_{\kappa \in K} \Delta_{\kappa} \sum_{i \in N} c_{i}^{\mathrm{g}} P_{i, t, \kappa}^{\mathrm{g}}
$$

In (14), $\psi_{t}$ is the load factor in stage $t$, and $\Delta_{\kappa}$ is the annual system operation time in condition $\kappa$, where the time duration of normal operation is much greater than the operation time under contingencies. Another possible objective in this work would be to include the cost of power losses with the cost of expansion. To consider only the minimisation of the cost of expansion, additional binary variables should be added to the piecewise linearisation, making the model more complex.

\subsection{Constraints}

The constraints of the MILP model for the multistage ACTNEP with simultaneous RPP considering security constraints are presented in this section, based on the AC load flow formulation (6)-(10) presented in Section 2. Since in (7)-(10) the voltage and currents magnitudes are squared, the variables change shown in (15) can be accomplished

$$
V_{i}^{\mathrm{sqr}}=V_{i}^{2} \text { and } I_{i j}^{\mathrm{sqr}}=I_{i j}^{2} ; \quad V_{i}^{\mathrm{sqr}} \geq 0 \text { and } I_{i j}^{\mathrm{sqr}} \geq 0
$$

Equations (16) and (17) represent the active and reactive power balances at bus $i$, stage $t$, scenario $\kappa$ in the ACTNEP problem. In (17), the variables $Q_{i, k i, y, t, \kappa}^{\text {shl }}$ and $Q_{i, i j, y, t, \kappa}^{\text {shl }}$ are calculated in (32) and
(33), while $Q_{i, r, t, \kappa}^{\text {shc }}$ is calculated in (41) and (42).

$$
\begin{aligned}
P_{i, t, \kappa}^{\mathrm{g}} & +\sum_{k i \in \Omega} \sum_{y \in Y} P_{k i, y, t, \kappa}-\sum_{i j \in \Omega} \sum_{y \in Y}\left(P_{i j, y, t, \kappa}+r_{i j, y, \kappa} I_{i j, y, t, \kappa}^{\mathrm{sqr}}\right) \\
& -G_{i}^{\mathrm{sh}} V_{i, t, \kappa}^{\mathrm{sqr}}=P_{i, t}^{\mathrm{d}} \\
Q_{i, t, \kappa}^{\mathrm{g}} & +\sum_{k i \in \Omega} \sum_{y \in Y}\left(Q_{k i, y, t, \kappa}+Q_{i, k i, y, t, \kappa}^{\mathrm{shl}}\right) \\
- & \sum_{i j \in \Omega} \sum_{y \in Y}\left(Q_{i j, y, t, \kappa}-Q_{i, i j, y, t, \kappa}^{\mathrm{shl}}+x_{i j, y, \kappa} I_{i j, y, t, \kappa}^{\mathrm{sqr}}\right)+B_{i}^{\mathrm{sh}} V_{i, t, \kappa}^{\mathrm{sqr}} \\
+ & \sum_{r \in R} Q_{i, r, t, \kappa}^{\mathrm{shc}}=Q_{i, t}^{\mathrm{d}} \quad \forall i \in N, \forall t \in T, \forall \kappa \in K
\end{aligned}
$$

Assuming that the voltage phase angle difference between buses $i$ and $j$, connected by line $i j$, is relatively small, (18) is a valid approximation.

$$
\sin \left(\theta_{i}-\theta_{j}\right) \cong \theta_{i}-\theta_{j}
$$

At this stage, a new parameter, $v_{i}$, is defined as an estimate of the voltage magnitude of bus $i$. The calculation of this parameter will be discussed later. After $v_{i}$ is known, $V_{i} V_{j}$ is approximated by $v_{i} v_{j}$ in (6). In addition, $v_{i}$ is used as an estimate of the voltage at bus $i$ in (8), and $V_{j}^{2}$ is approximated by $v_{j}^{2}$.

Considering these modifications, constraint (19) calculates the voltage magnitude drop, and (20) calculates the voltage phase angle difference in corridor $i j$ at stage $t$ in scenario $\kappa$, where phase shifters and off-nominal transformer turns ratios are not considered.

The parameter $v_{i, t, \kappa}$ is an estimate of the voltage at bus $i$ at stage $t$ in condition $\kappa$. The slack variables $f_{i j, t, \kappa}^{\mathrm{V}}$ and $f_{i j, t, \kappa}^{\theta}$, shown in (21) and (22), are equal to zero when there are (existing or new) lines in corridor $i j$ at stage $t$ in scenario $\kappa$; otherwise they are free. These variables are used to let the voltage magnitude and voltage phase angles vary freely in (19) and (20) when there are no (existing or new) lines in corridor $i j$ at stage $t$ in scenario $\kappa$, and to allow the calculation when there are lines. Note that, in (21) and (22), the summation will be equal to one if, and only if, an option $y$ with no lines is chosen (when $n_{i j, y, \kappa}=0$ ), and equal to zero otherwise. Also note that $(21)$ and $(22)$ are linear, since the absolute value function can be replaced by two inequalities, i.e. $|f| \leq \bar{f} \rightarrow-\bar{f} \leq f \leq \bar{f}$.

$$
\begin{gathered}
V_{i, t, \kappa}^{\mathrm{sqr}}-V_{j, t, \kappa}^{\mathrm{sqr}}-f_{i j, t, \kappa}^{\mathrm{V}} \\
=\sum_{y \in Y}\left[2\left(r_{i j, y, \kappa} P_{i j, y, t, \kappa}+x_{i j, y, \kappa} Q_{i j, y, t, \kappa}\right)+z_{i j, y, \kappa}^{2} I_{i j, y, t, \kappa}^{\mathrm{sqr}}\right] \\
v_{i, t, \kappa} v_{j, t, \kappa}\left(\theta_{i, t, \kappa}-\theta_{j, t, \kappa}\right)-f_{i j, t, \kappa}^{\theta}=\sum_{y \in Y}\left(x_{i j, y, \kappa} P_{i j, y, t, \kappa}-r_{i j, y, \kappa} Q_{i j, y, t, \kappa}\right)
\end{gathered}
$$

$$
\begin{gathered}
\left|f_{i j, t, \kappa}^{V}\right| \leq\left(\bar{V}^{2}-\underline{V}^{2}\right) \sum_{y \in Y \mid n_{i j, y, \kappa}=0} w_{i j, y, t} \\
\left|f_{i j, t, \kappa}^{\theta}\right| \leq 2 \bar{V}^{2} \bar{\theta} \sum_{y \in Y \mid n_{i j, y, \kappa}=0} w_{i j, y, t} \quad \forall i j \in \Omega, \forall t \in T, \forall \kappa \in K
\end{gathered}
$$

Constraints (23)-(30) represent the linearisation of the quadratic constraint that establishes the relationship between the active and reactive power flows of the equivalent circuit of option $y$ in corridor $i j$, the square of the voltage magnitude at the end of the corridor, and the square of the current flow magnitude of the equivalent circuit of option $y$ in corridor $i j$ for all stages and contingencies, where $\overline{\Delta S}_{i j, y, \kappa}=\bar{V} \bar{I}_{i j, y, \kappa} / L$ and $m_{i j, y, l, \kappa}^{\mathrm{s}}=(2 l-1)$ $\overline{\Delta S}_{i j, y, \kappa}$. The piecewise linearisation of the terms $P_{i j, y, t, \kappa}^{2}$ and $Q_{i j, y, t, \kappa}^{2}$ 
of the right-hand side of (23) is illustrated in Fig. 5.

$$
\begin{gathered}
v_{j, t, \kappa}^{2} I_{i j, y, t, \kappa}^{\mathrm{sqr}}=\sum_{l=1}^{L} m_{i j, y, l, \kappa}^{S} \Delta P_{i j, y, l, t, \kappa}+\sum_{l=1}^{L} m_{i j, y, l, \kappa}^{\mathrm{S}} \Delta Q_{i j, y, l, t, \kappa} \\
P_{i j, y, t, \kappa}^{+}-P_{i j, y, t, \kappa}^{-}=P_{i j, y, t, \kappa} \\
P_{i j, y, t, \kappa}^{+}+P_{i j, y, t, \kappa}^{-}=\sum_{l=1}^{L} \Delta P_{i j, y, l, t, \kappa} \\
Q_{i j, y, t, \kappa}^{+}-Q_{i j, y, t, \kappa}^{-}=Q_{i j, y, t, \kappa} \\
Q_{i j, y, t, \kappa}^{+}+Q_{i j, y, t, \kappa}^{-}=\sum_{l=1}^{L} \Delta Q_{i j, y, l, t, \kappa}
\end{gathered}
$$

$\forall i j \in \Omega, \forall y \in Y, \forall t \in T, \forall \kappa \in K$

$$
\begin{gathered}
0 \leq \Delta P_{i j, y, l, t, \kappa} \leq \overline{\Delta S}_{i j, y, \kappa} \\
0 \leq \Delta Q_{i j, y, l, t, \kappa} \leq \overline{\Delta S}_{i j, y, \kappa} \\
\forall i j \in \Omega, \forall y \in Y, l=1 \cdots L, \forall t \in T, \forall \kappa \in K \\
P_{i j, y, t, \kappa}^{+}, P_{i j, y, t, \kappa}^{-}, Q_{i j, y, t, \kappa}^{+}, Q_{i j, y, t, \kappa}^{-} \geq 0 \\
\forall i j \in \Omega, \forall y \in Y, \forall t \in T, \forall \kappa \in K
\end{gathered}
$$

Constraint (31) limits the current flow magnitude of the equivalent circuit of option $y$ in corridor $i j$ according to its operation state (i.e. if the option was chosen to be built or not, and if a contingency is considered or not) for all stages, normal operation, and contingency scenarios.

$$
0 \leq I_{i j, y, t, \kappa}^{\mathrm{sqr}} \leq w_{i j, y, t} \bar{I}_{i j, y, \kappa}^{2} \quad \forall i j \in \Omega, \forall y \in Y, \forall t \in T, \forall \kappa \in K
$$

The reactive power injection by the shunt element of the equivalent line of option $y$ in corridor $i j$, at bus $u(u=i$ or $u=j)$, for all stages and contingency scenarios is calculated by (32) and (33). Note that when $w_{i j, y, t}=0, Q_{u, i j, y, t, \kappa}^{\mathrm{sl}}=0$ in (33) and $\underline{V}^{2} \leq V_{u, t, \kappa}^{\mathrm{sqr}} \leq \bar{V}^{2}$ in (32). When $\quad w_{i j, y, t}=1, \quad \underline{V}^{2} b_{i j, y, \kappa}^{\mathrm{shl}} \leq Q_{u, i j, y, t, \kappa}^{\mathrm{shl}} \leq \bar{V}^{2} b_{i j, y, \kappa}^{\mathrm{shl}} \quad$ in $\quad$ (33) and

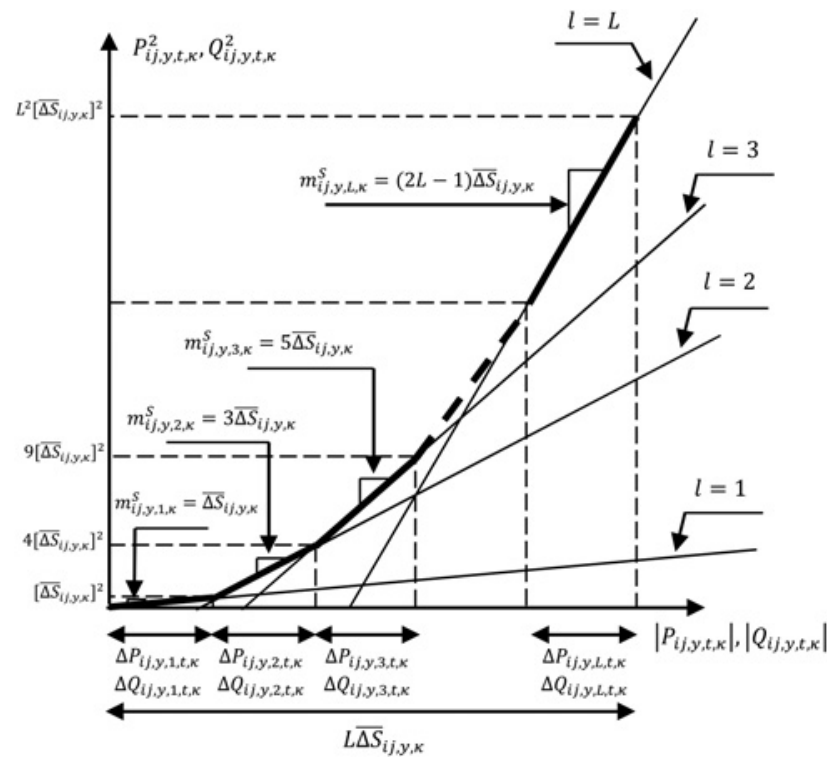

Fig. 5 Piecewise linearisation of $P_{i j, y, t, \kappa}^{2}$ and $Q_{i j, y, t, \kappa}^{2}$

$$
\begin{aligned}
Q_{u, i j, y, t, \kappa}^{\mathrm{shl}}= & V_{u, t, \kappa}^{\mathrm{sgr}} b_{i j, y, \kappa}^{\mathrm{shl}} \text { in }(32) . \\
& -\bar{V}^{2}\left(1-w_{i j, y, t}\right) b_{i j, y, \kappa}^{\mathrm{shl}} \leq Q_{u, i j, y, t, \kappa}^{\mathrm{shl}}-V_{u, t, \kappa}^{\mathrm{sqr}} b_{i j, y, \kappa}^{\mathrm{shl}} \\
& \leq-\underline{V}^{2}\left(1-w_{i j, t, y}\right) b_{i j, y, \kappa}^{\mathrm{shl}}
\end{aligned}
$$

$w_{i j, t, y} \underline{V}^{2} b_{i j, y, \kappa}^{\mathrm{shl}} \leq Q_{u, i j, y, t, \kappa}^{\mathrm{shl}} \leq w_{i j, y, t} \bar{V}^{2} b_{i j, y, \kappa}^{\mathrm{shl}}$

$\forall u \in N, \forall i j \in \Omega, \forall y \in Y, \forall t \in T, \forall \kappa \in K \mid u=i$ or $u=j$

Equation (34) determines that only one equivalent circuit option $y$ in corridor $i j$ can be chosen for each stage $t$. Constraint (35) limits the number of lines that can be added to corridor $i j$. Constraint (36) imposes that the number of lines in corridor $i j$ at stage $t$ must be greater than or equal to the number of lines in the same corridor at stage $t-1$, i.e. lines cannot be removed from one stage to the next.

$$
\begin{gathered}
\sum_{y \in Y} w_{i j, y, t}=1 \quad \forall i j \in \Omega, \forall t \in T \\
\sum_{y \in Y} y \cdot w_{i j, y, t} \leq \bar{n}_{i j} \quad \forall i j \in \Omega, \forall t \in T \\
\sum_{y \in Y} y \cdot w_{i j, y, t-1} \leq \sum_{y \in Y} y \cdot w_{i j, y, t} \quad \forall i j \in \Omega, \forall t \in T \mid t>1
\end{gathered}
$$

Constraint (34), together with (23) and (31), ensures that there will be no flow in a non-constructed line. Since (34) imposes that only one equivalent circuit option $y$ is chosen in each corridor, the flow in all lines that do not belong to the option that is chosen will be equal to zero. Suppose, for example, that in corridor $i j$ at stage $t=1$, there is one existing and two candidate lines. The options are $y=0, y=1$, and $y=2$, i.e. to construct zero, one, or two lines, respectively. If the model decides on option $y=1$, then $w_{i j, 0,1}=0$, $w_{i j, 1,1}=1$, and $w_{i j, 2,1}=0$. In this case, in (31), the current flow magnitudes in options $y=0$ and $y=2$ will be fixed at zero, and for option $y=1$, it will be limited by the maximum current of the chosen option (according to the contingency scenario). Finally, since the current magnitudes are fixed at zero for the options that are not chosen, the left hand side of (23) will be equal to zero for these options. Therefore, the active and reactive power flows will also be fixed at zero for options $y=0$ and $y=2$.

The limits for the voltage magnitudes are established by (37), while (38) limits the voltage phase angle at bus $i$, stage $t$, scenario $\kappa$. Equations (39) and (40) are the active and reactive power limits of the generators.

$$
\begin{gathered}
\underline{V}^{2} \leq V_{i, t, \kappa}^{\mathrm{sqr}} \leq \bar{V}^{2} \quad \forall i \in N, \forall t \in T, \forall \kappa \in K \\
-\bar{\theta} \leq \theta_{i, t, \kappa} \leq \bar{\theta} \quad \forall i \in N, \forall t \in T, \forall \kappa \in K \\
\underline{P}_{i, t}^{\mathrm{g}} \leq P_{i, t, \kappa}^{\mathrm{g}} \leq \bar{P}_{i, t}^{\mathrm{g}} \quad \forall i \in N, \forall t \in T, \forall \kappa \in K \\
\underline{Q}_{i, t}^{\mathrm{g}} \leq Q_{i, t, \kappa}^{\mathrm{g}} \leq \bar{Q}_{i, t}^{\mathrm{g}} \quad \forall i \in N, \forall t \in T, \forall \kappa \in K
\end{gathered}
$$

Similarly to (32) and (33), (41) and (42) provide the value of the reactive power injection by the VAr source $r$ connected at bus $i$ at stage $t$ in scenario $\kappa$.

$$
\begin{aligned}
&-\bar{V}^{2}\left(1-h_{i, r, t}\right) B^{\mathrm{shc}} \leq Q_{i, r, t, \kappa}^{\mathrm{shc}}-V_{i, t, \kappa}^{\mathrm{sqr}} B^{\mathrm{shc}} \\
& \leq-\underline{V}^{2}\left(1-h_{i, r, t}\right) B^{\mathrm{shc}} \\
& h_{i, r, t} \underline{V}^{2} B^{\mathrm{shc}} \leq Q_{i, r, t, \kappa}^{\mathrm{sc}} \leq h_{i, r, t} \bar{V}^{2} B^{\mathrm{shc}} \\
& \forall i \in N, \forall r \in R, \forall t \in T, \forall \kappa \in K
\end{aligned}
$$

Note that when $h_{i, r, t}=0, Q_{i, r, t, \kappa}^{\mathrm{shc}}=0$ in (42) and $\underline{V}^{2} \leq V_{i, t, \kappa}^{\mathrm{sqr}} \leq \bar{V}^{2}$ in 
(41). When $h_{i, r, t}=1, \quad \underline{V}^{2} B^{\text {shc }} \leq Q_{i, r, t, \kappa}^{\text {shc }} \leq \bar{V}^{2} B^{\text {shc }} \quad$ in $\quad$ (42) and $Q_{i, r, t, \kappa}^{\mathrm{shc}}=V_{i, t, \kappa}^{\mathrm{sqr}} B^{\mathrm{shc}}$ in (41).

Constraint (43) limits the number of VAr sources that can be connected at bus $i$ according to $\bar{m}_{i}$. The fencing constraint (44) imposes that the VAr sources must be installed in a particular order at a bus, i.e. source $r>1$ can only be installed if $r-1$ has already been installed. Constraint (45) imposes that if a VAr source was installed in stage $t-1$, it must not be removed in stage $t$

$$
\begin{gathered}
\sum_{r \in R} h_{i, r, t} \leq \bar{m}_{i} \quad \forall i \in N, \forall t \in T \\
h_{i, r, t} \leq h_{i, r-1, t} \quad \forall i \in N, \forall r \in R, \forall t \in T \mid r>1 \\
h_{i, r, t-1} \leq h_{i, r, t} \quad \forall i \in N, \forall r \in R, \forall t \in T \mid t>1
\end{gathered}
$$

Equation (46) provides a voltage phase angle reference for the system for each stage $t$ and scenario $\kappa$.

$$
\theta_{i, t, \kappa}=0 \quad \forall i \in N \mid i=\text { slack, } \forall t \in T, \forall \kappa \in K
$$

Constraints (47) and (48) represent the binary nature of the variables for the construction of lines and VAr sources, respectively.

$$
\begin{gathered}
w_{i j, y, t} \in\{0,1\} \quad \forall i j \in \Omega, \forall y \in Y, \forall t \in T \\
h_{i j, r, t} \in\{0,1\} \quad \forall i \in N, \forall r \in R, \forall t \in T
\end{gathered}
$$

Since the objective function (11) and all constraints (16), (17) and (19)-(46) in the model are linear, and there are binary variables (47) and (48), this is an MILP model. A two-step solution strategy is performed to obtain an estimate for $v_{i, t, \kappa}$. In the first step, the integrality of $w_{i j, y, t}$ and $h_{i j, r, t}$ is relaxed, and $v_{i, t, \kappa}=V^{\mathrm{n}}$; thus, the model (11), (16), (17) and (19)-(46) becomes a linear programming problem, with $w_{i j, y, t} \in[0,1]$ and $h_{i j, r, t} \in[0,1]$. Then, using the obtained values of $V_{i, t, \kappa}^{\mathrm{sqr}}, v_{i, t, \kappa}$ is set as $\sqrt{V_{i, t, \kappa}^{\mathrm{sqr}}}$ in (20) and (23), to be used in the second step. The second step consists of solving the MILP model (11), (16), (17) and (19)-(48) with the estimated value of $v_{i, t, \kappa}$. Note that in this second step, the voltages of all the buses are calculated in (19). The two-step solution strategy is illustrated in Fig. 6.

\section{Tests and results}

The proposed two-step strategy was implemented in the mathematical programming language AMPL [20] and solved using the commercial solver for optimisation problems CPLEX [21] (version 12.6.3.0, with default settings) on a computer with a 3.40 $\mathrm{GHz}$ Intel Core i7-4770 processor and $16 \mathrm{~GB}$ of RAM. Garver's 6-bus system [26] and a modified IEEE 118-bus system [27] were used to demonstrate the efficiency of the methodology. Complete data for both systems are available from [28]. An AC load flow method was used to analyse the accuracy of the results. For both tests, a planning horizon of 15 years was considered, divided into three planning stages of five years each. The operation horizons spanned from year 5 until year $10\left(\Upsilon_{1}^{i}=5\right.$ and $\left.Y_{1}^{f}=10\right)$ for the first stage, from year 10 until year $15\left(\Upsilon_{2}^{i}=10\right.$ and $\left.Y_{2}^{f}=15\right)$ for the second stage, and from year 15 until year $25\left(\Upsilon_{2}^{2}=15\right.$ and $\left.\Upsilon_{2}^{f}=25\right)$ for the third stage. In addition, the number of blocks of the piecewise linearisation was $L=10$, the annual discount rate was $\alpha=10 \%$, and $\psi_{t}=0.6$ for all stages. It was also assumed that $\bar{\theta}=90^{\circ}$, and the annual time duration was $8760 \mathrm{~h}$. The VAr sources consisted of modules of $B^{\text {shc }}=0.2$ p.u., with a cost, $c_{i}^{\text {sh }}$, of MUS $\$ 0.05$ each. The voltage magnitude limits were $\underline{V}=0.95$ p.u. and $\bar{V}=1.05$ p.u.
Initialise $v_{i, t, \kappa}$ as the nominal voltage of the system $V^{n}$ only in (20) and (23);

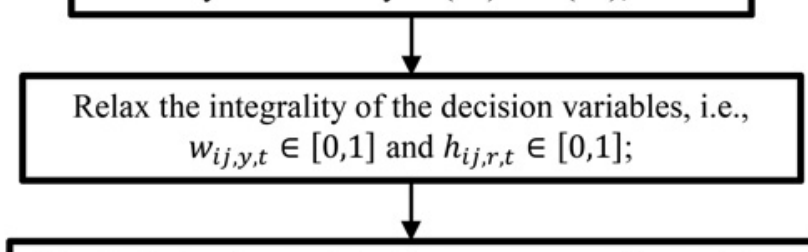

Step 1: Solve the resulting linear programming problem (11), (16), (17) and (19)-(46);

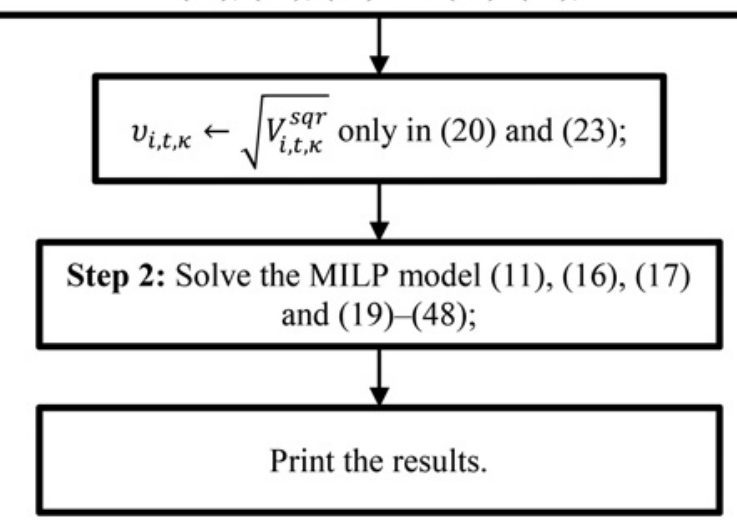

Fig. 6 Two-step solution strategy

\subsection{Garver's 6-bus system}

The original data for this system are presented in [26]. The system had 15 transmission corridors. It was assumed that each corridor could admit a maximum of three lines, and a maximum of three VAr sources could be installed at each load bus $\left(\bar{m}_{i}=3\right)$. Nine scenarios were considered for each stage, one considering normal operation and the remaining eight considering outages of lines in corridors $1-2,1-4,1-5,2-3,2-4,2-6,3-5$, and 4-6 (one outage per contingency scenario). The problem consisted of 7,782 linear equality constraints, 13,185 linear inequality constraints, 198 binary variables, and 36,816 continuous variables. Table 1 shows the planning results for the three stages considered. The result obtained for the 6-bus system is also illustrated in Fig. 7.

Both the line transmission and VAr planning are presented, together with the total cost of expansion. The computational time needed to solve the problem was 6.6 minutes. To verify the accuracy of the results, an AC load flow was executed for each scenario $\kappa$ in stage $t$, using the solution of the proposed model. For the AC load flow calculations, the planning solutions for each stage were fixed, along with the active generation and voltage magnitudes of each generation bus, with the exception of bus 1 for which only the voltage magnitude and angle reference were fixed.

The operation results (obtained with the AC load flow) for the three stages are shown in Table 2. The total energy losses, cost of losses, energy generation, cost of operation, and the error in the values of the cost of operation - between the solution obtained with the proposed model and the AC load flow - are presented for each stage. Note that from Table 2, the costs of operation of the proposed linearised model are very close to the exact operation values of the system, which were obtained with the AC load flow.

Table 1 Planning results for the 6-bus system

\begin{tabular}{lccc}
\hline Stage & New lines & New VAr sources & Cost, MUS\$ \\
\hline 1 & $(2-3),(3-5),(4-6)$ & $(4) \times 2$ & 43.53 \\
2 & $(4-6)$ & $(2)$ & 11.58 \\
3 & $(2-6),(3-5),(4-6)$ & - & 19.15 \\
total & & & 74.26 \\
\hline
\end{tabular}




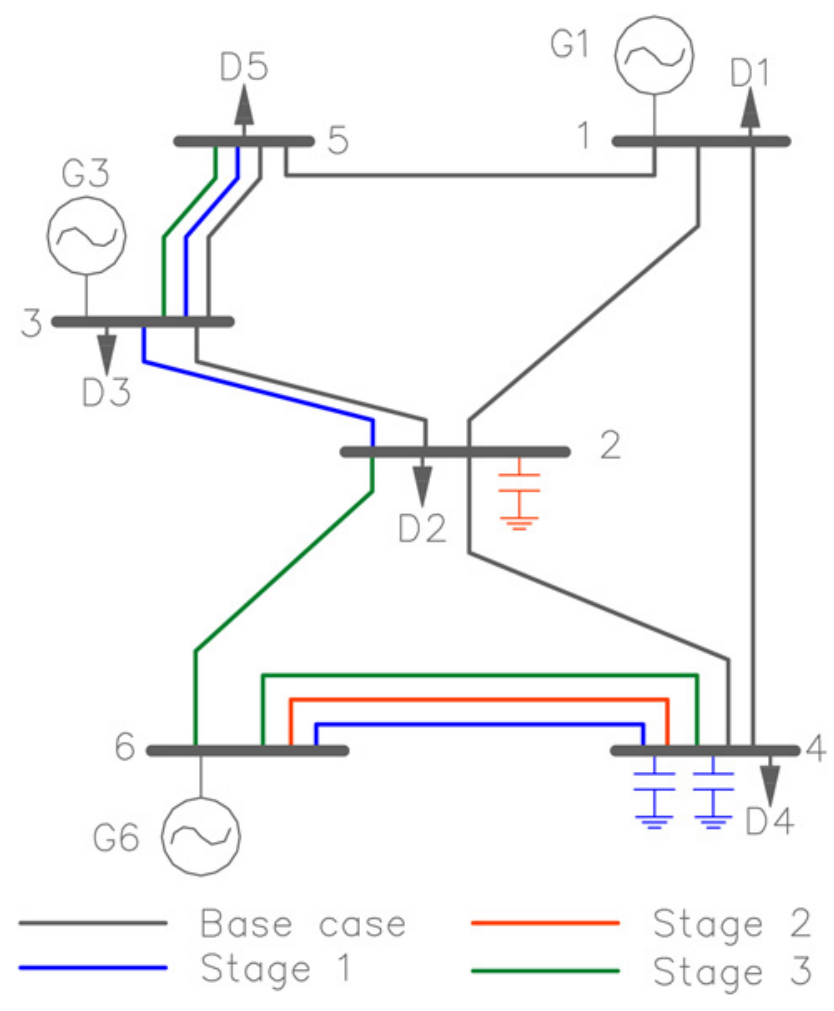

Fig. 7 Solution for the Garver's system

This shows that the proposed model represents the operation of the system with good accuracy.

Table 3 shows the results of the maximum and average errors for the voltage magnitudes and voltage phase angles of the buses, and the active and reactive power flows in the lines, considering all stages and contingency scenarios.

The results show that the proposed planning methodology provides an accurate solution for the ACTNEP with simultaneous RPP, with minimal errors. Analysing the results of the AC load flow, it was verified that all constraints of the ACTNEP problem were fulfilled. A second case was then analysed without the RPP in the TNEP, i.e. considering only the expansion of lines. The solution obtained had a total cost of MUS\$ 401.32 (cost of expansion: MUS\$ 90.42; cost of operation: MUS\$ 310.90), with a cost of losses of MUS\$ 10.85. For this system, when considering the TNEP with simultaneous RPP, the cost of expansion was reduced due to postponing the installation of a line in corridor 4-6 from stage 1 to stage 3 and not installing a line in corridor 2-3 in stage 3. This shows the importance of considering RPP simultaneously with TNEP.

Table 2 Operation results for the 6-bus system

\begin{tabular}{lccccc}
\hline Stage & $\begin{array}{c}\text { Total } \\
\text { energy } \\
\text { losses, } \\
\text { GWh }\end{array}$ & $\begin{array}{c}\text { Cost of } \\
\text { losses, } \\
\text { MUS\$ }\end{array}$ & $\begin{array}{c}\text { Total energy } \\
\text { generation, } \\
\text { GWh }\end{array}$ & $\begin{array}{c}\text { Cost of } \\
\text { operation, } \\
\text { MUS\$ }\end{array}$ & $\begin{array}{c}\text { Error in the } \\
\text { cost of } \\
\text { operation, } \\
\%\end{array}$ \\
\hline 1 & 463.78 & 4.37 & 12447.46 & 87.48 & 0.01 \\
2 & 565.34 & 3.30 & 16543.58 & 90.34 & 0.11 \\
3 & 1297.26 & 3.82 & 41242.86 & 133.54 & 0.01 \\
total & 2326.38 & 11.49 & 70233.90 & 311.36 & 0.03 \\
\hline
\end{tabular}

Table 3 Comparison of the results for the 6-bus system

\begin{tabular}{lcccc}
\hline Variable & $V_{i, t, \kappa^{\prime}} \%$ & $\theta_{i, t, \kappa^{\prime}}{ }^{\circ}$ & $P_{i j, y_{,}, \kappa^{\prime}} \%$ & $Q_{i j, y_{,}, \kappa^{\prime}} \%$ \\
\hline max. error & 0.524 & 2.369 & 1.142 & 2.205 \\
avg. error & 0.033 & 0.287 & 0.258 & 0.212 \\
\hline
\end{tabular}

Table 4 Planning results for the 6-bus system using the DC model and the reinforcements needed

\begin{tabular}{|c|c|c|c|c|c|}
\hline \multirow[t]{2}{*}{ Stage } & \multicolumn{2}{|c|}{$\begin{array}{l}\text { Solution for the DC } \\
\text { model }\end{array}$} & \multicolumn{3}{|c|}{$\begin{array}{l}\text { Solution for the } \mathrm{DC} \text { model with } \\
\text { reinforcements }\end{array}$} \\
\hline & New lines & $\begin{array}{l}\text { Cost, } \\
\text { MUS\$ }\end{array}$ & Lines & $\begin{array}{l}\text { New VAr } \\
\text { sources }\end{array}$ & $\begin{array}{l}\text { Cost, } \\
\text { MUS\$ }\end{array}$ \\
\hline 1 & $\begin{array}{c}(2-3),(3-5) \\
(4-6)\end{array}$ & 43.46 & $\begin{array}{c}(2-3),(3-5), \\
(4-6)\end{array}$ & $(4) \times 2$ & 43.53 \\
\hline 2 & $(4-6)$ & 11.57 & $(3-5),(4-6)$ & (2) & 19.30 \\
\hline 3 & $\begin{array}{c}(2-6) \times 2 \\
(3-5)\end{array}$ & 19.15 & $\begin{array}{l}(2-6) \times 2 \\
(4-6)\end{array}$ & - & 21.54 \\
\hline cost & & 74.18 & \multicolumn{2}{|c|}{ total cost } & 84.37 \\
\hline
\end{tabular}

A third case was considered to show the advantage of using the proposed model instead of first performing the expansion of lines using the DC model and then considering the RPP, as it is proposed in most of works about TNEP. In the first part of this test, the DC model for the TNEP problem was solved; then, the solution (new lines) was fixed, and the proposed model was used to find the solution for the RPP, without allowing the construction of new lines. The solution of the DC model is presented in Table 4.

Using the solution of the DC model and then performing the RPP without adding new lines was not enough to find a feasible solution considering the AC operation. This indicates that the solution of the DC model must be reinforced, i.e. more lines must be added to the system for the AC operation to be feasible.

The second part of this case fixed the solution of the DC model, i.e. the lines of the solution of the DC model cannot be removed, and then the proposed ACTNEP model was used to find a feasible solution for the problem, allowing the construction of new lines or anticipating the line construction of the DC model solution. The final result of the DC model reinforced by the proposed AC model is also shown in Table 4.

Starting from the solution for the DC model, the proposed model indicates that the line in corridor (3-5) must be constructed in stage 2 , instead of stage 3 , and a new line must be added in corridor (4-6). The solution of the RPP is the same as presented in Table 1. The cost of expansion was MUS\$ 84.37, as opposed to MUS\$ 74.26 when the proposed ACTNEP model with RPP was considered to solve the problem directly.

\subsection{Modified IEEE 118-bus system}

A modified version of the IEEE 118-bus system [27] was the second case analysed. In order to create congestion, the capacities of the lines were reduced, as in [14], and some of the lines were removed from the corridors. The cost of adding a line to corridor $i j$ was also estimated as in [14]. For this case, in 25 out of the 179 transmission corridors, a maximum of two lines could be added [28], and a maximum of two VAr sources could be installed at each load bus $\left(\bar{m}_{i}=2\right)$. Eleven scenarios were considered for each stage, one considering normal operation and the remaining ten considering contingency scenarios corresponding to the outages of lines in corridors $1-3,15-17,25-27,60-61,38-65,64-65$, $77-78,86-87,80-99$, and 12-117 [14]. The problem consisted of 92,628 linear equality constraints, 64,238 linear inequality constraints, 801 binary variables, and 278,646 continuous variables. Table 5 shows the planning results for the three stages considered. The result obtained for the 118-bus system is also illustrated in Fig. 8.

Table 5 Planning results for the 118-bus system

\begin{tabular}{lccc}
\hline Stage & New lines & New VAr sources & Cost, MUS\$ \\
\hline 1 & $(8-9),(9-10),(99-100)$, & $(45),(93),(95)$ & 29.22 \\
& $(12-117)$ & & \\
2 & $(38-37),(77-78)$ & $(1),(93),(95)$ & 3.74 \\
3 & $(26-30),(93-94)$ & $(1),(11),(13),(44),(94)$ & 6.92 \\
total & & & 39.88 \\
\hline
\end{tabular}




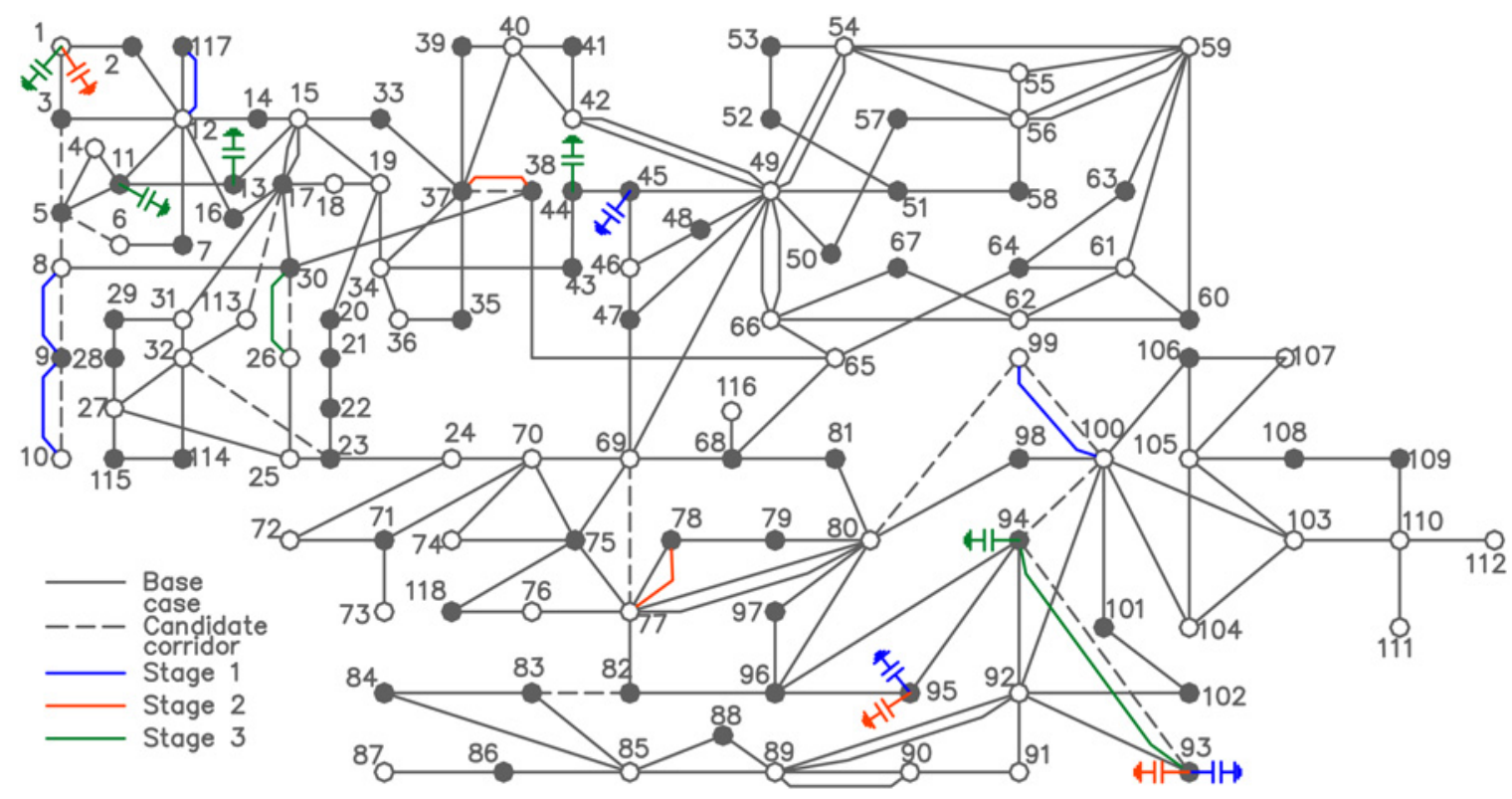

Fig. 8 Solution for the 118-bus system

The computational time needed to solve the problem was 55 minutes. The operation results, obtained with the AC load flow, for the three stages are shown in Table 6. The total energy losses, cost of losses, energy generation, cost of operation, and the error in the values of the cost of operation - between the solution obtained with proposed model and the AC load flow - are presented for each stage. Again, the costs of operation of the proposed linearised model were very close to the values of the real operation of the system, obtained with the AC load flow, with errors lower than $0.11 \%$. As in the first case, the results of the $\mathrm{AC}$ load flow show that the solution obtained by the ACTNEP model fulfilled the constraints of the problem.

Table 7 shows the results of the maximum and average errors for the voltage magnitudes and voltage phase angles of the buses, and the active and reactive power flows in the lines, considering all stages and contingency scenarios.

Then, a second case was analysed without the RPP in the TNEP. The solution obtained presented a total cost of MUS\$1050.79 (cost of expansion: MUS\$ 42.39; cost of operation: MUS\$ 1008.40), with a cost of losses of MUS\$ 2.31. Again, the results for the TNEP with simultaneous RPP were better than those for the case without RPP. In this second case, when considering the TNEP simultaneously with RPP, the cost of expansion was reduced due to installing a line in corridor 93-94 in stage 3 instead of installing a line in corridor 92-93 in stage 2.

In addition, a third case was considered, first solving the DC model for the TNEP problem, fixing the expansion solution, and

Table 6 Operation results for the 118-bus system

\begin{tabular}{lccccc}
\hline Stage & $\begin{array}{c}\text { Total } \\
\text { energy } \\
\text { losses, } \\
\text { GWh }\end{array}$ & $\begin{array}{c}\text { Cost of } \\
\text { losses, } \\
\text { MUS\$ }\end{array}$ & $\begin{array}{c}\text { Total energy } \\
\text { generation, } \\
\text { GWh }\end{array}$ & $\begin{array}{c}\text { Cost of } \\
\text { operation, } \\
\text { MUS\$ }\end{array}$ & $\begin{array}{c}\text { Error in the } \\
\text { cost of } \\
\text { operation, } \\
\%\end{array}$ \\
\hline 1 & 333.53 & 0.31 & 71752.06 & 337.78 & 0.07 \\
2 & 1094.00 & 0.64 & 103465.11 & 302.43 & 0.11 \\
3 & 4733.48 & 1.39 & 250424.15 & 368.36 & 0.03 \\
total & 6161.01 & 2.34 & 425641.32 & 1008.57 & 0.07 \\
\hline
\end{tabular}

Table 7 Comparison of the results for the 118-bus system

\begin{tabular}{lcccc}
\hline Variable & $V_{i, t, \kappa_{1}} \%$ & $\theta_{i, t, \kappa^{\prime}}{ }^{\circ}$ & $P_{i j, y, t, \kappa^{\prime}} \%$ & $Q_{i j, y, t, \kappa^{\prime}} \%$ \\
\hline max. error & 0.140 & 1.363 & 1.340 & 1.452 \\
avg. error & 0.003 & 0.192 & 0.097 & 0.055 \\
\hline
\end{tabular}

then using the proposed model to find the solution for the RPP without allowing the construction of new lines. The solution of the DC model is presented in Table 8 .

Once again, performing the RPP considering the solution of the DC model, without adding new lines, was not enough to find a feasible solution considering the AC operation. Therefore, the solution of the DC model must be reinforced.

In the second part of this case, the solution of the DC model was fixed, and then the proposed ACTNEP model was used to find a feasible solution for the problem, allowing the construction of new lines or anticipating the construction of the lines of the solution of the DC model. The final result of the DC model reinforced by the proposed AC model is also shown in Table 8.

Using the solution for the DC model, the proposed model indicates that the line in corridor (38-37) must be constructed in stage 2, instead of stage 3 . The solution for this case was the same as the one reported in Table 5. Note, however, that the DC model did not find this solution directly, i.e. the proposed model changed the expansion plan of the DC model in order to get a feasible solution.

\section{Conclusion}

An MILP model for solving the problem of transmission network expansion simultaneously with RPP has been presented. The proposed model considers multistage expansion and security

Table 8 Planning results for the 118-bus system using the DC model and the reinforcements needed

\begin{tabular}{|c|c|c|c|c|c|}
\hline \multirow[t]{2}{*}{ Stage } & \multicolumn{2}{|c|}{$\begin{array}{c}\text { Solution for the DC } \\
\text { model }\end{array}$} & \multicolumn{3}{|c|}{$\begin{array}{l}\text { Solution for the DC model with } \\
\text { reinforcements }\end{array}$} \\
\hline & New lines & $\begin{array}{l}\text { Cost, } \\
\text { MUS\$ }\end{array}$ & Lines & $\begin{array}{l}\text { New VAr } \\
\text { sources }\end{array}$ & $\begin{array}{l}\text { Cost, } \\
\text { MUS\$ }\end{array}$ \\
\hline 1 & $\begin{array}{c}(8-9),(9-10) \\
(99-100) \\
(12-117)\end{array}$ & 29.13 & $\begin{array}{c}(8-9),(9-10), \\
(99-100) \\
(12-117)\end{array}$ & $\begin{array}{l}\text { (45), (93), } \\
\text { (95) }\end{array}$ & 29.22 \\
\hline 2 & $(77-78)$ & 1.08 & $\begin{array}{l}(38-37) \\
(77-78)\end{array}$ & $\begin{array}{l}(1),(93) \\
(95)\end{array}$ & 3.74 \\
\hline 3 & $\begin{array}{l}(26-30), \\
(38-37), \\
(93-94)\end{array}$ & 8.47 & $\begin{array}{l}(26-30) \\
(93-94)\end{array}$ & $\begin{array}{c}(1),(11), \\
(13),(44), \\
(94)\end{array}$ & 6.92 \\
\hline cost & & 38.68 & \multicolumn{2}{|c|}{ total cost } & 39.88 \\
\hline
\end{tabular}


constraints, while the objective function minimises the total cost of expansion and operation. The use of an MILP model guarantees convergence to optimality using classical optimisation techniques. The AC operation of the network is represented by a linearised model that takes into account reactive power, bus voltage magnitudes, and power losses. Tests comparing the calculated steady-state operation points to those given by an AC load flow method demonstrated the precision of the proposed formulation.

Tests carried out with Garver's 6-bus system and a modified IEEE 118-bus system demonstrated the efficiency of the proposed solution technique for the transmission network expansion problem. The results showed that the proposed model is precise and, therefore, can provide more adequate planning solutions than the DC model when the AC operation is considered. In addition, better expansion and generation plans can be found by considering RPP simultaneously with the TNEP. Results demonstrate that considering simultaneously the installation of VAr sources, along with the construction of lines, postponed and/or eliminated the necessity of investing in new lines and reduced the cost of generation. The proposed model also provided better solutions than the conventional practice of solving the TNEP problem using the DC model and then performing the RPP.

\section{Acknowledgments}

This work was supported by CNPQ, CAPES, and São Paulo Research Foundation (FAPESP), under grant 2014/23741-9.

\section{References}

1 Latorre, G., Cruz, R.D., Areiza, J.M., et al.: 'Classification of publications and models on transmission expansion planning', IEEE Trans. Power Syst., 2003, 18, (2), pp. 938-946

2 Romero, R., Monticelli, A., Garcia, A., et al.: 'Test systems and mathematical models for transmission network expansion planning', IEE Pro. Gener. Transm. Distrib., 2002, 149, (1), pp. 27-36

3 Lee, C.W., Ng, S., Zhong, J., et al.: 'Transmission expansion planning from past to future'. Proc. IEEE PES Power Systems Conf. Expo., Atlanta, United States, October 2006, pp. 257-265

4 Molina, J.D., Rudnick, H.: 'Transmission of electric energy: a bibliographic review', IEEE Latin America Trans., 2010, 8, (3), pp. 245-258

5 Zhang, W., Li, F., Tolbert, L.M.: 'Review of reactive power planning: objectives, constraints, and algorithms', IEEE Trans. Power Syst., 2007, 22, (4), pp. $2177-2186$

6 Hemmati, R. Hooshmand, R., Khodabakhshian, A. 'State-of-the-art of transmission expansion planning: Comprehensive review', Renew. Sustain. Energy Rev., 2013, 23, pp. 312-319
7 Rider, M.J., Garcia, A.V., Romero, R.: 'Power system transmission network expansion planning using AC model', IET Gener. Transm. Distrib., 2007, 1, (5), pp. 731-742

8 Rahmani, M., Rashidinejad, M., Carreño, E.M., et al.: 'Efficient method for AC transmission network expansion planning', Electr. Power Syst. Res., 2010, 80, pp. 1056-1064

9 Hooshmand, R.-A., Hemmati, R., Parastegari, M.: 'Combination of AC transmission expansion planning and reactive power planning in the restructured power system', Energy Convers. Manage., 2012, 55, pp. 26-35

10 Bent, R., Toole, G.L., Berscheid, A.: 'Transmission network expansion planning with complex power flow models', IEEE Trans. Power Syst., 2012, 27, (2), pp. 904-912

11 Mahmoudabadi, A., Rashidinejad, M.: 'An application of hybrid heuristic method to solve concurrent transmission network expansion and reactive power planning', Int. J. Electr. Power Energy Syst., 2013, 45, pp. 71-77

12 Glover, F., Kochenberger, G.: 'Handbook of Metaheuristics' (Kluwer Academic Publishers, 2003, 1st edn. 2003)

13 Zhang, H., Heydt, G.T., Vittal, V., et al.: 'Transmission expansion planning using an AC model: formulations and possible relaxations'. Proc. IEEE PES General Meeting, San Diego, United States, July 2012, pp. 1-8

14 Zhang, H., Heydt, G.T., Vittal, V., et al.: 'An improved network model for transmission expansion planning considering reactive power and network losses', IEEE Trans. Power Syst., 2013, 28, (3), pp. 3471-3479

15 Akbari, T., Bina, M.T.: 'A linearized formulation of AC multi-year transmission expansion planning: A mixed-integer linear programming approach', Electr. Power Syst. Res., 2014, 114, pp. 93-100

16 Taylor, J.A., Hover, F.S.: 'Linear relaxations for transmission system planning', IEEE Trans. Power Syst., 2011, 26, (4), pp. 2533-2538

17 Asadamongkol, S., Eua-Arporn, B.: 'Transmission expansion planning with AC model based on generalized Benders decomposition', Int. J. Electr. Power Energy Syst., 2013, 47, pp. 402-407

18 Taylor, J.A., Hover, F.S.: 'Conic AC transmission system planning', IEEE Trans. Power Syst., 2013, 28, (2), pp. 952-959

19 Jabr, R.A.: 'Optimization of AC transmission system planning', IEEE Trans. Power Syst., 2013, 28, (3), pp. 2779-2787

20 Fourer, R., Gay, D.M., Kernighan, B.W.: 'AMPL: A modeling language for mathematical programming' (Brooks/Cole-Thomson Learning, 2003, 2nd edn. 2003)

21 'IBM ILOG CPLEX'. Available at http://www-01.ibm.com/software/integration/ optimization/cplex-optimization-studio, accessed 25 November 2015

22 Farivar, M., Low, S.H. 'Branch flow model: relaxations and convexification-Part I', IEEE Trans. Power Syst., 2013, 28, (3), pp. 2554-2564

23 Grainger, J.J., Stevenson, W.D.: 'Power System Analysis' (McGraw-Hill, 1994, 1st edn. 1994)

24 Franco, J.F., Rider, M.J., Lavorato, M., et al.: 'Optimal conductor size selection and reconductoring in radial distribution systems using a mixed-integer LP approach', IEEE Trans. Power Syst., 2013, 28, (1), pp. 10-20

25 Ross, S.A., Westerfield, R.W., Jaffe, J.: 'Corporate finance' (McGraw-Hill/Irwin, 2010, 9th edn. 2010)

26 Garver, L.L.: 'Transmission network estimation using linear programming', IEEE Trans. Power Appar. Syst., 1970, 89, (7), pp. 1688-1697

27 'Illinois Institute of Technology, Electrical and Computer Engineering Department - IEEE 118-bus System Data'. Available at http://motor.ece.iit.edu/ Data/Gastranssmion 118 14test.xls, accessed 10 December 2015

28 'LaPSEE Power System Test Cases Repository'. Available at http://www.feis. unesp.br/\#!/lapsee, accessed 10 December 2015 